\title{
Ultra Wideband Monocycle pulse generation using SRD and Coupled line band pass filter with reduced ringing level
}

\author{
Amol M Sapkal ${ }^{1}$, Dr.Nisha Sarwade ${ }^{2}$, Bhaskar Shelkod ${ }^{3}$ \\ M.Tech Scholar, Electrical Engineering Department, VJTI, Mumbai, India ${ }^{1}$ \\ Assistant Professor, Electrical Engineering Department, VJTI, Mumbai, India ${ }^{2}$ \\ Scientist-B, MED-II, SAMEER, Mumbai, India ${ }^{3}$
}

\begin{abstract}
This paper presents simple ultra wideband monocycle pulse generator by SRD and coupled line band pass filter element values for $0.5 \mathrm{~dB}$ equal ripple. Proposed design deals with generating Gaussian pulse from square waveform as a input then using high order coupled line band pass filter we get ultra wideband monocycle pulse of $6 \mathrm{mv}$ (p-p) of amplitude and pulse width of 500psec We eliminates use of lumped elements (Inductor, capacitor) and external bias circuit for generation of Gaussian pulse, due to which ringing level is reduced. Simulation is carried out using Advanced design systems (ADS).
\end{abstract}

Keywords: UWB, SRD, Coupled line band pass Filter, ADS.

\section{INTRODUCTION}

Ultra-wideband is a wireless technology that can operate in $\mathrm{MHz}-\mathrm{GHz}$ frequency range consists of narrow pulse having pulse width of picosec-nanosec[2]. Ultra wide band and narrow band can be differentiate in terms of bandwidth ,information content, Circuit complexity, antenna, front-end, analog and digital baseband Signals[10]. UWB has characteristics to Penetrate objects, Hence it can be used in Medical Imaging applications, such as cancer detection.

In this paper we use UWB Pulse generator in medical Imaging system for application of early breast cancer detection in which array of UWB antenna is used which operate in an extremely wideband frequency spectra, 3.1$10.6 \mathrm{GHz}$ bandwidth at $-41.3 \mathrm{dBm} / \mathrm{MHz}$ for medical applications which is restricted by Federal Communication Commission of US(FCC)[1][5]. There are various types of Pulses used for UWB transmission ;Gaussian pulse, Gaussian monocycle, Rayleigh monocycle, RZ Manchester but out of which Gaussian monocycle pulse[2] i.e. higher order Gaussian pulse preferred because its spectrum meets into FCC mask and spectrum doesn't contains low frequency components and dc hence widely used in many applications.

In this paper a simple low cost ultra wideband pulse generator is designed using step recovery diodes (SRD) and coupled band pass filter i.e. we take square waveform as input signal then using SRD it is converted in Gaussian pulse $2.5 \mathrm{v}$ in amplitude and $1 \mathrm{nsec}$ in pulse duration which is shaped by $5^{\text {th }}$ order coupled band pass filter which gives narrow Gaussian monocycle pulse of $6 \mathrm{mv}(\mathrm{p}-\mathrm{p})$ and pulse width of 500 psec.

The circuit consists of two major parts: Gaussian pulse generator using SRD illustrates in section 2 and micro strip coupled band pass filter illustrate in section3[5][6].Due to use of highly ordered coupled band pass filter it insures that the output of Gaussian monocycle pulse meet mask spectrum restricted by FCC.

\section{GAUSSIAN PULSE GENERATOR}

Firstly time domain rectangular pulses with $5 \mathrm{v}$ peak is input square signal with rise time $0.269 \mathrm{nsec}$ applied to the Gaussian Pulse Generator Then SRD is forward biased and charged up. The charges stored in step recovery diode are extracted during the next digital high half cycle of driving source, resulting in a current pulse with a rise time equivalent to the "snap time" of the diode[4]. The balanced single reflection short stub behind further shapes the output into a Gaussian pulse. Further output taken across load resistance RL[18].

Fig. 2.(a) is a circuit diagram of the Gaussian pulse generator that is designed with two Metallic's MMD-805 Step Recovery Diode(SRD) Model MODEL D_805 has following specifications ( $\mathrm{IS}=500.0 \mathrm{E} 15, \mathrm{~N}=1.3, \mathrm{RS}=0.13$, CJ0 $04.55 \mathrm{E} 12, \quad \mathrm{VJ}=0.5, \quad \mathrm{M}=0.235, \quad \mathrm{XTI}=3.0, \quad \mathrm{EG}=1.12$, $\mathrm{BV}=60, \mathrm{IBV}=10 \mathrm{E}-6, \mathrm{TT}=30 \mathrm{E}-9)$

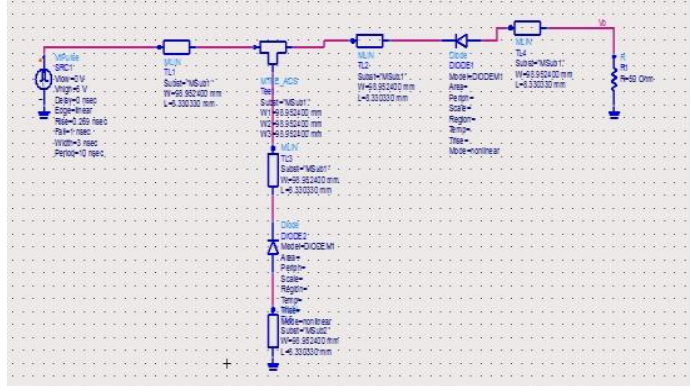

(a) 

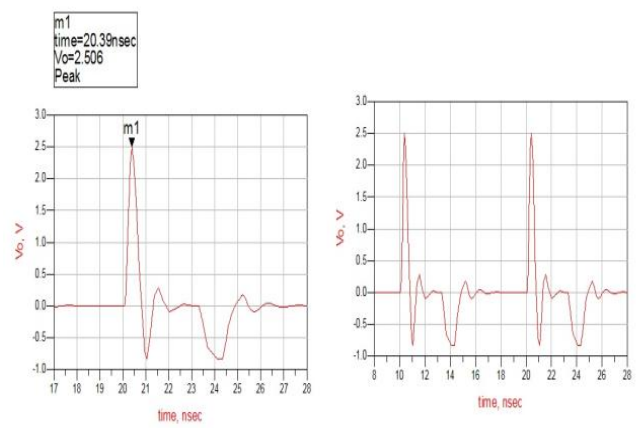

(b)

Fig.2(a) Gaussian Pulse generator (b) Time domain simulated results of Gaussian Pulse generator

Transient simulation was carried out by using Advanced Design System(ADS) with time range 0nsec- 100nsec with 1 nsec each step size.

The substrate used is FR4 with dielectric constant of 4.4 and thickness of $0.016 \mathrm{~mm}$ and height is $1.5 \mathrm{~mm}$,loss tangent is 0.02 having characteristics impedance is 50 ohm. Time domain results of the Gaussian Pulse Generator in Fig 2.(b) were measured using ADS. The measured Gaussian pulse has a pulse amplitude of $2.5 \mathrm{~V}$, with pulse width of $1 \mathrm{~ns}$. The measured second pulse due to parasitic capacitance of SRD is at approximately $140 \mathrm{mV}$ which is slightly smaller than that of the simulated results

\section{FIFTH ORDER BANDPASS FILTER}

Fig.3 (a) shows the filter structure proposed in this paper known as parallel coupled filter[8]. The strips are arranged parallel close to each other, so that they are coupled with certain coupling factors. We use the following equations for designing the parallel-coupled filter[11].

The band pass frequency ranges from $3.1-10.6 \mathrm{GHz}$ i.e. Bandwidth is given by $(W 2-W 1) / W o$ where $W o$ given $\operatorname{as} \sqrt{ }(W 2 * W 1)$.

Bandwidth $(\mathrm{BW})=5.732 \mathrm{GHz}$

$\mathrm{Z} 0 \mathrm{~J} 1=\frac{\sqrt{(\pi * B W)}}{(2 * g 1)}$
$\mathrm{Z} 0 \mathrm{Jn}=\frac{\pi * B W}{2 \sqrt{g n-1 * g n}}$

$\mathrm{g} 0, \mathrm{~g} 1, . .$, gn can be taken from $0.5 \mathrm{~dB}$ Equal-Ripple LowPass Filter Prototypes table and $\mathrm{BW}$ is the relative bandwidth, $\mathrm{Jn}$ is the characteristic admittance of $\mathrm{J}$ inverter and $\mathrm{Zo}$ is the characteristic admittance of the connecting transmission line.

Further can calculate the characteristic impedances of even-mode and odd-mode of the parallel-coupled microstrip transmission line, as follows[11]

$\mathrm{Z} 0 \mathrm{e}=\mathrm{Zo}\left[1+\mathrm{JZo}+(\mathrm{JZo})^{\wedge} 2\right]$

$\mathrm{Z} 0 \mathrm{e}=\mathrm{Zo}\left[1-\mathrm{JZo}+(\mathrm{JZo})^{\wedge} 2\right]$

for $\mathrm{n}=1,2 \ldots \mathrm{N}$

Where $\mathrm{N}$ is order of the filter here $\mathrm{N}$ is equal to the 5
Further can calculate the characteristic impedances of even-mode and odd-mode of the parallel-coupled microstrip transmission line, as shown in Table 3.1

The coupled filter Calculations using even and odd impedances are given in Table.3.1

Table 3.1. Coupled line filter Characteristic

\begin{tabular}{|c|c|c|c|c|}
\hline $\mathrm{N}$ & Gn & $\mathrm{ZoJn}$ & $\mathrm{Z} 0 \mathrm{e}(\Omega)$ & $\mathrm{Z0e}(\Omega)$ \\
\hline 1 & 1.7058 & 1.0941 & 164.55 & 55.147 \\
\hline 2 & 1.2296 & 1.41 & 219.905 & 78.905 \\
\hline 3 & 2.5408 & 1.1553 & 174.5 & 58.97 \\
\hline 4 & 1.2296 & 1.1553 & 174.5 & 58.97 \\
\hline 5 & 1.7058 & 1.41 & 219.905 & 78.905 \\
\hline
\end{tabular}

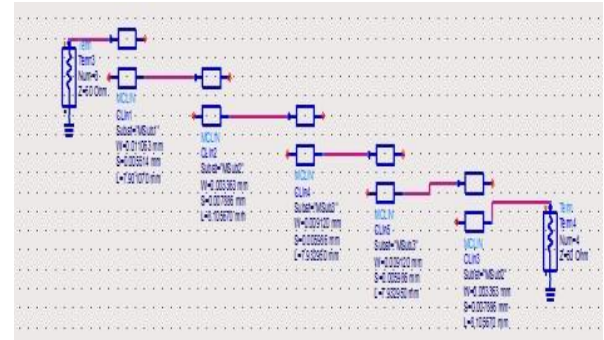

(a)
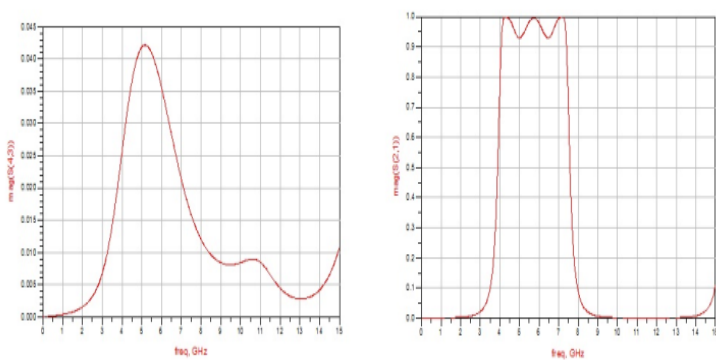

(b)

Fig.3.Fifth order coupled line BPF (a) schematic diagram (b) Simulated S21 of the Fifth order band pass filter in ADS

\section{UWB MONOCYCLE PULSE GENERATOR}

Results obtained from Gaussian Pulse generator and the fifth derivative reflection network were then integrated to develop UWB pulse and circuit diagram in ADS using the modeled digital source is shown in Fig.4 (a) in which transient simulation is carried out

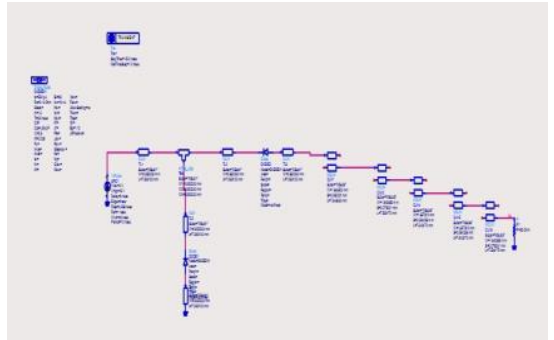

(a) 

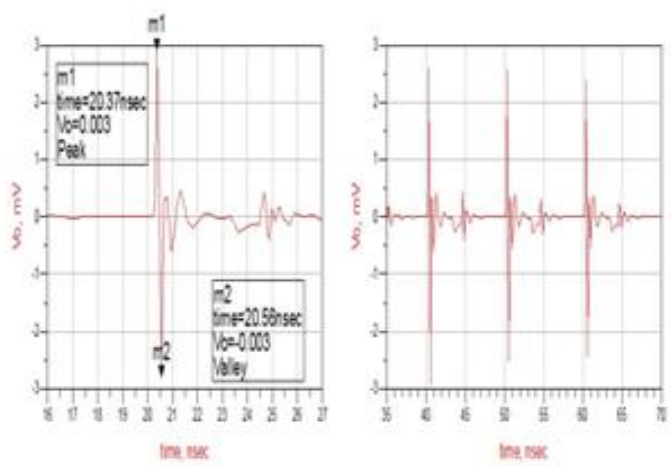

(b)

Fig.4.UWB Monocycle pulse generator (a) schematic diagram (b) Time domain simulated results in ADS

In Fig.4.(a) is a fifth order Gaussian pulse is generated via simulation using ADS with slight ringing dying off at the end of the pulse[8][9]. The ringing is due to the parasitic capacitance and inductance of the SRD, as well as the characteristic of the reflection network to induce slight ringing. The pulse width without taking the ringing into consideration is $500 \mathrm{ps}$ and the peak-to-peak voltage of the pulse is $6 \mathrm{~m} \mathrm{~V}$.

\section{CONCLUSION}

This paper designed a new ultra-wideband (UWB) monocycle pulse generator. This pulse generator uses a simple SRD Gaussian pulse generator and fifth order band pass filter to generate the monocycle pulse directly. In this design work , use of Lumped element is prohibited i.e. capacitor and inductor due to which Package parasitic capacitance and inductance cannot distort the output waveform, hence obtained output has low ringing effect.

Another benefits of this design is it uses only one square pulse generating circuit hence no additional biasing DC source is not needed for this design work

\section{REFERENCES}

[1]. Werfelli, H.; Chaoui, M.; Ghariani, H.; Lahiani, M., "Design of a pulse generator for UWB communications," Systems, Signals \& Devices (SSD), 2013 10th International Multi-Conference on , vol., no., pp.1,6, 18-21 March 2013.

[2]. Orlenko, O.A., "UWB pulse generators," Ultrawideband and Ultrashort Impulse Signals (UWBUSIS), 2012 6th International Conference on , vol., no., pp.75,77, 17-21 Sept. 2012

[3]. Shahid, Z.; Khan, S.; Alam, A.H.M.Z., "Sub-nano seconds UWB pulse shaping with reduced ringing phenomenon," Computer and Communication Engineering (ICCCE), 2012 International Conference on , vol., no., pp.617,620, 3-5 July 2012.

[4]. Anfu Zhu; Fu Sheng; Zhang, Anxue, "An implementation of step recovery diode-based UWB pulse generator," Ultra-Wideband (ICUWB), 2010 IEEE International Conference on, vol.2, no., pp.1,4, 20-23 Sept. 2010.

[5]. Cemin Zhang; Fathy, A.E., "Reconfigurable Pico-Pulse Generator for UWB Applications," Microwave Symposium Digest, 2006. IEEE MTT-S International , vol., no., pp.407,410, 11-16 June 2006

[6]. Pavel protiva, jan mrkvica, jan machac, "Universal Generator of Ultra-Wideband Pulses "Radioengineering, vol. 17, 2008

[7]. Dong-Ho Kim; Gyeong-Nam Bang; Park, Chongdae, "Design and characteristics of high order derivative gaussian pulse generator for DS-UWB," Microwave Conference, 2006. APMC 2006. AsiaPacific , vol., no., pp.1110,1113, 12-15 Dec. 2006

[8]. Low, Z.N.; Cheong, J.H.; Law, C.L., "Novel low cost higher order derivitive Gaussian pulse generator circuit," Communications
Systems, 2004. ICCS 2004. The Ninth International Conference on , vol., no., pp.30,34, 7-7 Sept. 2004.

[9]. Jeongwoo Han; Cam Nguyen, "A new ultra-wideband, ultra-short monocycle pulse generator with reduced ringing," Microwave and Wireless Components Letters, IEEE, vol.12, no.6, pp.206,208, June 2002.

[10]. David Barras, Frank Ellinger and Heinz Jäckel , "Comparison between -Wideband and Narrowband Transceivers" , FCC authorization of Ultra Wideband technology, 2000

[11]. D.M. Pozar, "Microwave engineering", 2nd edition, 1998 JohnWiley \& Sons.

[12]. ADS Simulation Tool 2011 